\title{
Earth saved from disaster!
}

\section{There were high hopes that comet Swift-Tuttle might be on collision course with the Earth, but it now seems that enthusiasts for destruction will have to wait for another ill-omened body.}

THE profound satisfaction that comes from contemplating the destruction of our entire planet is presumably an infinitely multiplied form of the satisfaction we get from watching the bad guy's car, at the end of a movie, speed over the edge of a cliff, plunge into a rocky chasm, and burst into flames. We are alarmed, for example, at the thought that human activity might be changing the climate of the Earth enough to cause the demise of our species, but at the same time is there not a certain thrill in the idea that we might have become so technologically capable that we have the power to extinguish a whole planet?

For the connoisseur of catastrophe, however, global warming is a protracted and uncertain venture. Already there are moves afoot to curtail our collective output of greenhouse gases into the atmosphere, and it seems quite possible that global warming may be averted before anything genuinely destructive comes of it. Global destruction, if it is to have wholesale appeal, should be abrupt, and should have a degree of cinematic splendour. This is why, surely, there is so much continuing fascination with the extinction at the Cretaceous/Tertiary boundary and the end of the dinosaurs. A slow dwindling of the dinosaur population, perhaps because of changing climate, is unappcalingly tame, but if an asteroid or comet ploughed into the Earth and sent up dust that darkened the skies and snuffed out a substantial fraction of all life on the globe, then we have a screenplay our imaginations can latch onto. Of course, we are still here to talk about the dinosaurs, and there is an extra vicarious pleasure to be gained from thinking that we - in the form of some small and adaptable ancestral mammal were smart enough to survive the destruction that did for the dim-witted and lumbering reptiles.

In science-fiction books and movies that portray an Earth in the grip of some imminent death-threat, there is generally at least one smart and adaptable creature who, mocked at first (just as the first rodents were no doubt scorned endlessly by the dinosaurs), comes through with a brilliant scheme to save the world - excluding those who mocked, of course. Earlier this year, it was suggested in the United States that a good way to adapt the defence industry to modern times would be to have the ingenious weapons designers work on saving the Earth; their expertise could be used for a massive effort against global warming or, more excitingly, they could be asked to find ways to make nuclear weapons effective against external threats, notably the impacts of comets or asteroids. These suggestions were largely discounted as either special pleading for the defence industry or foolish romancing by scientists who had not yet noted that the world's economy was in recession. But of course it has always been the fate of the farsighted to be shunned, and anyway astronomers could prove that a collision between the Earth and a chunk of celestial rock large enough to be damaging must, by the laws of probability, happen one day.

In happy fulfilment of these dire thoughts, comet Swift-Tuttle entered onto the scene in September. The purely scientific interest in this matter was that Swift-Tuttle, first observed in 1862, had been predicted to return in 1981, but never showed up. But Brian Marsden, of the Harvard-Smithsonian Center for Astrophysics, had also suggested the possibility of a later return, on the grounds that a comet seen in 1737 might have been Swift-Tuttle on an earlier circuit. The 1981 return was predicated on an orbit governed purely by gravitation, but to tie the 1737 and 1862 apparitions together, Marsden had to throw into his calculations some non-gravitational effects: comets eject gas when they are heated by the Sun, and these jets can be enough to perturb the orbit. With an estimate of non-gravitational effects included, Swift-Tuttle's orbit would, according to Marsden, bring it back to the inner solar system in 1992. In September an amateur comet-watcher in Japan spotted an object in Ursa Major which the experts soon declared to be Swift-Tuttle.

So much for the science. What made Swift-Tuttle exciting was that its orbit, recalculated by Marsden with the help of the new sighting, will bring it very close to the Earth on its next return, in 2126. In fact it will miss the Earth by about 19 days, or 15 million miles, which is close enough to be interesting but hardly the stuff of which movies are made. Why then did this modest prediction turn into newspaper stories foretelling the possible terrestrial impact, a century and a half hence, of a celestial body comparable to the one that wiped out the dinosaurs? What prompted the editors of Newsweek to run a cover story entitled "Doomsday Science", complete with artist's rendition of Swift-Tuttle bearing fierily down on an innocent, pearly-blue Earth?

When he reported his calculation in the IAU Circulars (a telegram system by which astronomers quickly alert each other to important observations and events), Marsden noted that the chance of a collision of SwiftTuttle with the Earth, in 2126, was about one in ten thousand. But he also observed that the non-gravitational influences on the orbit, sufficient to shift the predicted return by 11 years, from 1981 to 1992 , were inherently unpredictable, and that a mere 15 day correction to the calculated perihelion of Swift-Tuttle would be enough to put it on a collision course with Earth.

It is tempting to think that if the time of perihelion can change by 11 years, then a change of merely 15 days is somehow a likely possibility, but of course the opposite is true: if the moment of perihelion is random in the interval plus or minus 11 years around the predicted return, then the chance of Swift-Tuttle arriving on any one chosen date is indeed about 1 in 10,000 . The Newsweek article noted the low probability of collision but went on to list in enthusiastic detail the wondrous horrors that would ensue: an explosive force of 100 million megatons of TNT, a plume of vaporized stone, buildings and even trees bursting into flame, rain as caustic as the acid in a car battery, and other phenomena marvellous to imagine.

Unfortunately for catastrophists, none of this seems at all likely, and there is little chance that reformed weapons designers will be able to show off their talents by designing missiles that would intercept Swift-Tuttle and push it off track by means of artificially induced non-gravitational forces. IAU Circular number 5672 however offered a double tease, briefly raising hopes of a fatal collision. First there was a suggestion that Swift-Tuttle, because it suffers unusually large non-gravitational perturbations, might also be susceptible to fragmentation; if a shower of large comet chunks filled the orbital path, Earth's chances of intercepting a dangerously large rock would be correspondingly larger. Second on Circular 5672 was an observational note that Swift-Tuttle indeed seemed to have split into pieces - three secondary nuclei were spotted, travelling en famille with the parent body. Gratifyingly, the odds of doomsday were on the up again.

Alas, IAU Circular 5673 squelched even these hopes. It reported that the observations of secondary nuclei were due to optical reflections in the telescope, and that SwiftTuttle was therefore still a single comet plodding to its non-rendezvous with the Earth. Despite all the hopes pinned on it, Swift-Tuttle is resolutely refusing to be the agent of wild destruction. Too bad!

David Lindley 